\title{
Diagnostics of Coronal Heating in Solar Active Regions
}

\author{
A. Fludra \\ CCLRC Rutherford Appleton Laboratory, Didcot, OX11 OQX, UK
}

J. Ireland

L3com. Analytics Corp., NASA GSFC, Greenbelt, MD 20771, USA

\begin{abstract}
We study the relationship between EUV spectral line intensities and the photospheric magnetic field in solar active regions, using magnetograms from SOHO-MDI and EUV spectra of the Fe XVI $360.8 \AA$ line $\left(2 \times 10^{6} \mathrm{~K}\right)$ and the O V $629.7 \mathrm{~A}$ line $(220,000 \mathrm{~K})$ from the Coronal Diagnostic Spectrometer on $\mathrm{SOHO}$, recorded for several active regions. We overlay and compare spatial patterns of the $\mathrm{O} \mathrm{V}$ emission and the magnetic flux concentrations, with a $4^{\prime \prime} \times 4^{\prime \prime}$ spatial resolution, and search for a relationship between the local $\mathrm{O} \mathrm{V}$ line intensity and the photospheric magnetic flux density in each active region. While this dependence exhibits a certain amount of scatter, it can be represented by a power law fit. The average power index from all regions is $0.7 \pm 0.2$. Applying static loop models, we derive the dependence of the heating rate on the magnetic flux density, $E_{h} \propto B^{0.8}$, and compare it to the dependence predicted by the coronal heating models.
\end{abstract}

\section{Introduction}

One of the fundamental questions in solar physics is identifying the mechanism that heats the solar corona. While a large theoretical effort has resulted in many models, it is not known yet which of those processes operates on the sun. Observational constraints on the coronal heating models can be obtained from relationships between the photospheric magnetic flux and intensities of EUV spectral lines and X-rays emitted from the solar atmosphere. Active regions provide the best data to study this topic, due to strong concentrations of magnetic field, a large range of magnetic flux density, and strong EUV emission. Previous work (e.g., Gurman et al., 1974; Schrijver 1987; Fisher et al. 1998) found power-law relations between the total magnetic flux, $\Phi$, and total intensities of a few chromospheric, transition region and coronal emission lines in active regions, e.g., $I_{X-r y} \propto \Phi^{1.2}$.

Data from the Coronal Diagnostic Spectrometer (CDS) on SOHO together with the SOHO/MDI magnetograms provide an excellent material to make a substantial advance in the study of the coronal heating. Using four spectral lines from the CDS synoptic data set: He I $584.3 \mathrm{~A}\left(2 \times 10^{4} \mathrm{~K}\right), \mathrm{O}$ V $629.7 \mathrm{~A}$ $\left(2.2 \times 10^{5} \mathrm{~K}\right), \mathrm{Mg}$ IX $368.06 \mathrm{~A}\left(9.5 \times 10^{5} \mathrm{~K}\right)$, and Fe XVI $360 \mathrm{~A}\left(2.5 \times 10^{6} \mathrm{~K}\right)$, 
Fludra and Ireland (2002a,b; 2003b) found power laws between the total line intensities, integrated over an active region area, and the total unsigned magnetic flux $\Phi$. For the two best diagnostics, the coronal Fe XVI $360.8 \mathrm{~A}$ line and the transition region O V $629.7 \mathrm{~A}$ line, they find that the total Fe XVI intensity is proportional to $\Phi^{1.2}$, and the total O V intensity is proportional to $\Phi^{0.78}$. Since the total unsigned photospheric magnetic flux in the $50-500 \mathrm{G}$ range is nearly proportional to the magnetic area (Fludra and Ireland, 2003b), a large part of the global dependence, $I_{t o t} \propto \Phi^{\alpha}$, can be explained by the variation of the active region area. However, there exists an underlying local $I-B$ dependence that can be used as a diagnostic of the coronal heating.

Fludra and Ireland (2003a) show for the first time that the equation describing how the observed total line intensity integrated over an active region area arises from the magnetic field, can be approximated by a Laplace integral:

$$
I_{\infty} / H_{0}=\int_{0}^{\infty} I(\phi) \exp (-\beta \phi) d \phi
$$

where $\beta$ is the slope of the histogram of the unsigned magnetic flux density $\phi$ above $50 \mathrm{G}, H_{0}$ is the normalization of the histogram, and $I_{\infty}$ is the intensity that would arise if the histogram extended from $\phi=0$ to infinity $\left(I_{\infty}\right.$ can be determined iteratively). They use this property to solve an inverse problem and derive a function relating the line intensity from individual loops to the photospheric magnetic flux density at their footpoints. They propose a simple model in which the intensity of a coronal line Fe XVI 360.8 A in an individual coronal loop is proportional to the footpoint magnetic flux density to the power of $\delta$ :

$$
I(\phi)=Q_{1} L^{\lambda} \phi^{\delta}
$$

and explore how well the value of delta is constrained by the observations. For the coronal line Fe XVI, they obtain $\delta=1.3$, where $1.0<\delta<1.6$ with $90 \%$ confidence.

Our simulations (Fludra and Ireland 2003b) using static loop models show that the intensity of the coronal Fe XVI line is proportional to $P^{2}$, where $P$ is pressure, for loop-top temperatures in the range 2.0-2.5 million $\mathrm{K}$. Thus, using scaling laws, we have $I_{F e} \propto E_{h}^{12 / 7}$. Combining this with $I_{F e} \propto \phi^{1.3}$, we obtain $E_{h} \propto \phi^{0.75}$.

In this paper we extend the analysis to another spectral line, O V 629.7 $\AA$, which is emitted at transition region temperatures $\left(2.2 \times 10^{5} \mathrm{~K}\right)$ and thus its intensity in loops that reach coronal temperatures is always proportional to pressure (Martens et al. 2000). This is a convenient property for the study of the coronal heating because for static loops it relates the intensity and the volumetric heating rate $E_{h}, I_{l o o p} \propto E_{h}^{6 / 7}$.

First, we assess whether we can use the Laplace transform to invert Equation 1 for the OV line intensity in the same way as Fludra and Ireland (2003a) did for the Fe XVI line intensity. We find that the parameter delta is not too well constrained by the global relationship $(\delta=0.8 \pm 0.3$ for all 45 active regions considered, and $\delta=0.7 \pm 0.5$ for 26 active regions without sunspots). Therefore, in this paper we will use a spatially-resolved comparison between the OV 
intensity and the magnetic field, instead of the global relationship of integrated quantities. However, that global relationship gives us an indication of the dependence of the intensity on the average active region size: when we approximate an average loop length $L{ }_{v}$ in each region as the square root of the projected area of the coronal Fe XVI emission, the goodness-of-fit criterion $\chi^{2}$ is minimized for $L_{v}^{\lambda}, \lambda=-0.5 \pm 0.1$.

\section{Comparison of Spatially Resolved Data}

We adopt the following procedure of aligning the CDS OV rasters with the MDI magnetograms: a simulated intensity image in the OV $629.7 \AA$ line is calculated from the magnetogram by assuming a model $I=|B|^{\delta}$ at each magnetogram pixel, for $\delta=0.5$. This simulated intensity map is smeared out using a spatial point spread function (PSF) representing the PSF of the CDS (a 2D gaussian with $\mathrm{FWHMX}=1.7^{\prime \prime}$, FWHMY $=8^{\prime \prime}$ ). The observed CDS OV raster and the simulated OV intensity map are interpolated to a finer step of $0.5^{\prime \prime}$ and aligned by performing a $2 \mathrm{D}$ cross-correlation. After the co-alignment, both images are rebinned to a coarser pixel size of $4^{\prime \prime} \times 4^{\prime \prime}$. Assuming the relationship $I=Q_{0} \phi^{\delta}$, we vary $\delta$ from 0 to 1.2 with a step of 0.1 . For each value of $\delta, Q_{0}$ is calculated to minimize $\chi^{2}$ that compares the $\log _{10}$ of observed and simulated intensities for all pixels with the magnetic flux density greater than $20 \mathrm{G}$. The optimum $\delta$ is that which gives the global minimum of $\chi^{2}$. Fig. 1a shows the comparison of simulated and observed $\mathrm{OV}$ intensities for one of the active regions, for the optimum value of $\delta$. Fig. 1b compares the observed OV intensities with a 5point boxcar average of the simulated intensities which significantly reduces the scatter. Clearly, on small spatial scales $\left(4^{\prime \prime} \times 4^{\prime \prime}\right.$ pixels $)$ the relationship between the line intensity and the magnetic field is more complex and has greater scatter than it would appear from spatially integrated $I-\Phi$ plots (e.g., Fludra and Ireland 2003b). We attribute the scatter to a combination of statistical noise and a distribution of loop lengths inside active regions.

For six active regions we have obtained the following values of $\delta: 0.5,0.7$, $0.6,0.6,0.9,0.7$, each with a range of \pm 0.1 . The uncertainty reflects a range of values of $\delta$ that give $\chi^{2}$ close to its minimum value. The average value is $0.7 \pm 0.2$. The dependence on $L$ cannot be determined for individual active regions because we do not measure loop lengths inside active regions.

The intensity of a transition region line emitted from a static loop is:

$$
I_{O V}=c E_{h}^{6 / 7} L^{5 / 7} \int_{T 1}^{T 2} G(T) d T
$$

where $L$ is the loop length and $G(T)$ is the line emissivity. Combining the relationship $I_{O V} \propto \phi^{0.7}$ derived from the observations, with Equation 3 we obtain the heating rate $E_{h} \propto \phi^{\beta}, \beta=0.8 \pm 0.2$. This is close to the value of $\beta=0.75$ obtained for the Fe XVI line in Section 1 . The power index of 0.8 in this relationship is comparable to the index of 1.0 obtained by Schrijver and Aschwanden (2002) from simulations of solar and stellar soft X-ray emission, and to the index of 0.9 obtained by Yashiro and Shibata (2001) based on the data from the Yohkoh Soft X-ray Telescope. 

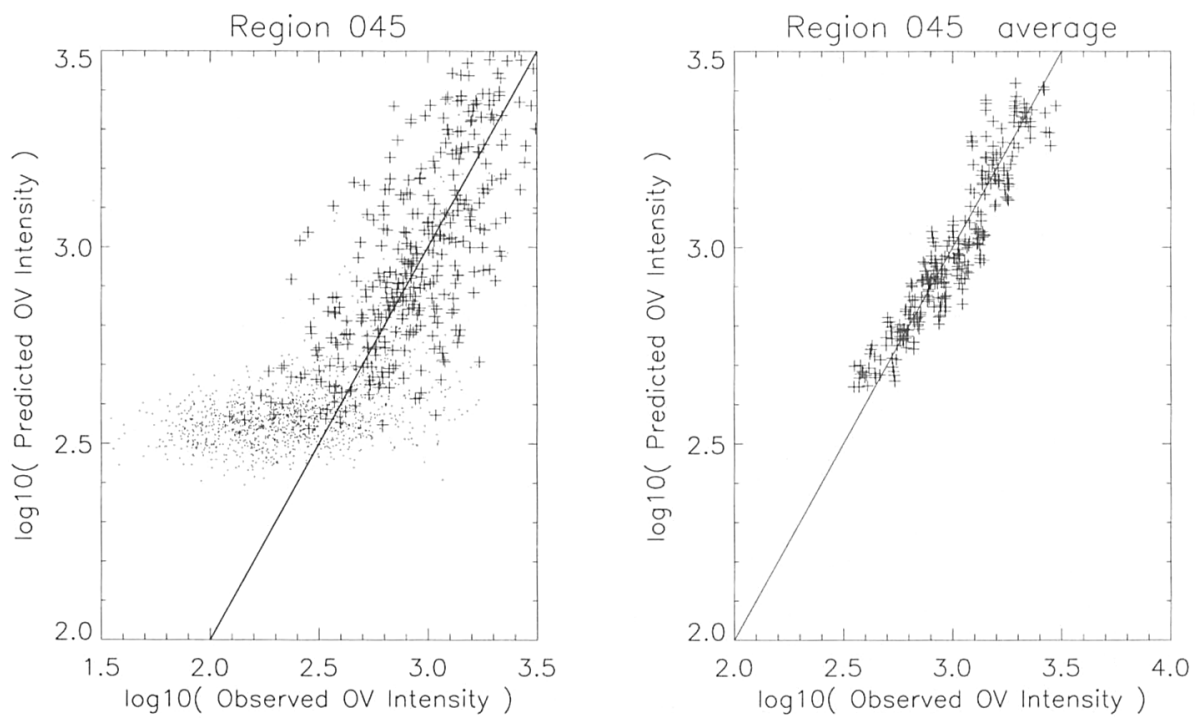

Figure 1. (a) A comparison of observed and simulated OV intensities $\left(I \propto|\phi|^{\delta}\right)$ for the optimum value of $\delta$ (left panel), for one selected active region. Crosses denote pixels with magnetic flux density $\phi>20 \mathrm{G}$, used to calculate $\chi^{2}$. Dots denote pixels with a weak magnetic field $(\phi<20 \mathrm{G})$. (b) A comparison of the observed OV intensities with a 5 -point boxcar average of the simulated intensities (right panel). The continuous line is an $y=x$ relationship.

\section{The Heating Mechanism}

To see how this result constrains the heating mechanism, we refer to Mandrini et al. (2000); in this work, the authors review many heating models, characterizing the heating rate as $E_{h} \propto B_{c}^{\beta} L^{\lambda}$, where $B_{c}$ is the coronal magnetic field. Among the 22 models reviewed by Mandrini et al. (2000), two models predict $E_{h}$ proportional to $B$. The first one (model 4 in Table 5 of Mandrini et al. 2000) is a direct current (DC) model considering magnetic reconnection in current sheets. The second model is in the alternating current (AC) class, with high-frequency boundary excitation (referred to as model 21 in Mandrini et al. 2000). These two models are closest to our result. Most other models predict $\beta$ equal to $-1,0,1.5$, and 2 . Our result, $E_{h} \propto B^{0.8}$, is in contrast with most DC coronal heating models which predict the volumetric heating rate proportional to $B^{2}$.

Acknowledgments. This work was supported by the UK Particle Physics and Astronomy Research Council. SOHO is a project of international cooperation between ESA and NASA. 


\section{References}

Fisher, G.H., Longcope, D.W., Metcalf, T.R., \& Pevtsov, A.A. 1998, ApJ, 508, 885

Fludra, A., \& Ireland, J. 2003a, A\&A, 398, 297

Fludra, A., \& Ireland, J. 2003b, In: "The Future of Cool-Star Astrophysics", Eds. A. Brown, G. M. Harper, \& T. R. Ayres. Proceedings of 12 th Cambridge Workshop on Cool Stars, Stellar Systems, \& the Sun, p. 220 (http://origins.Colorado.EDU/cs12/proceedings/oral/tuesday/ fludraproc.html)

Fludra, A., Ireland, J., del Zanna, G., \& Thompson, W. T. 2002a, Adv. Space Res., Vol. 29, Issue 3, p. 361

Fludra, A., \& Ireland, J., 2002b, In: SOLMAG 2002. Proceedings of the Magnetic Coupling of the Solar Atmosphere Euroconference and IAU Colloquium 188, ESA Publications Division, ESA SP-505, p. 405

Fludra, A., \& Ireland, J., In: Proceedings of the SOHO 11 Symposium on From Solar Min to Max: Half a Solar Cycle with SOHO, ESA Publications Division, 2002, ESA SP-508, p. 267

Gurman, J.B., Withbroe, G.L., \& Harvey, J.W. 1974, Solar Phys., 34, 105

Mandrini, C.H., Demoulin, P., \& Klimchuk, J.A. 2000, ApJ, 530, 999

Martens, P.C.H., Kankelborg, C.C., \& Berger, T.E. 2000, ApJ, 537, 471

Schrijver, C.J., 1987, A\&A, 180, 241

Schrijver, C.J., \& Aschwanden, M.J. 2002, ApJ, 566, 1147

Yashiro, S., \& Shibata, K. 2001, ApJ, 550, L113 